\title{
Covid-19: out-of-hours providers are drafted in to manage non-urgent patients in community
}

\author{
Elisabeth Mahase
}

The BMJ

Regional NHS leaders in England have been ordered to immediately organise a primary care management service to care for covid-19 patients who "do not require immediate admission" to hospital.

In a letter sent on 8 March, seen by The BMJ, NHS England and NHS Improvement's strategic incident director for coronavirus, Keith Willett, has ordered regional primary care and public health directors to set up a 24 hour, seven day a week service to manage patients in the community. This service should be delivered by an out-of-hours provider, and every part of England must be covered by Tuesday 10 March, the letter said.

The service will be used to manage patients who are deemed well enough to be isolated at home, with active monitoring for people who are at high risk of developing severe illness, and advice to those not deemed high risk on what to do if their illness deteriorates.

The decree marks a change from previous practice, which required every patient who tested positive to the virus to be admitted to hospital even if they had only mild symptoms.

Each provider could receive up to $£ 20000$ ( $€ 23000$; \$26 000) to meet the "costs around infrastructure and mobilisation of capacity" or a proportionate sum when a single provider stepped up to cover the area usually covered by several providers. "An indicative tariff of $£ 100$ per patient for the service applies, to be reviewed in light of arrangements for home visits," Willett's letter added.

The community service will initially be delivered through telephone or online channels, with the potential for home visits to be included later. But the letter said that if "gaps in provision remain, we potentially have the opportunity to bring in a provider of digital first primary care to support the response."

Patients who test positive for the virus will be assessed to ensure they can be managed at home, before their details are passed to the out-of-hours provider. Anyone needing immediate care that cannot be given in the community, and people who cannot safely isolate themselves at home, will be admitted to hospital.

But people deemed to be at high risk (over 60s and people of any age with cardiovascular disease, chronic respiratory disease, diabetes, or severe immunosuppression) who are experiencing a mild illness and can be managed in the community will be told to isolate themselves at home and will be contacted daily by phone to check their symptoms.

The letter advises that patients "remain in isolation until 5 days after resolution of symptoms, unless [they are a] healthcare worker or work with high risk groups, in which case require one negative sample 5 days after resolution of symptoms before return to work.'

People with mild illness and not in a high risk group will be told to isolate themselves at home and will be given health advice on how to identify deterioration. All patients managed at home will be given a phone number to call if they feel more unwell.

The letter said that the community service should be provided by a nurse and GP team and that all clinical information should be recorded and transferred to the patient's general practice.

Providers must also provide regular situation reports, including confirmed numbers of patients cared for under the service, numbers of patients who deteriorate, and numbers of patients admitted to and discharged from the service. 